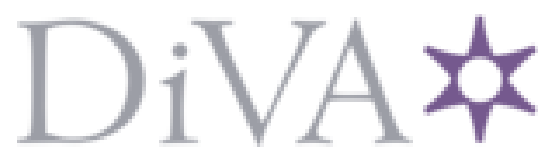

http://www.diva-portal.org

\title{
Postprint
}

This is the accepted version of a paper presented at 13th International Conference on Probabilistic Methods Applied to Power Systems (PMAPS); Durham, UK, 7-10 July 2014.

Citation for the original published paper:

Wallnerström, C., Hilber, P., Söderström, P., Saers, R., Hansson, O. (2014)

Potential of dynamic rating in Sweden.

In:

N.B. When citing this work, cite the original published paper.

Permanent link to this version:

http://urn.kb.se/resolve?urn=urn:nbn:se:kth:diva-147995 


\section{Potential of dynamic rating in Sweden}

\author{
C. J. Wallnerström, P. Hilber \\ $\mathrm{KTH}$ \\ Stockholm, Sweden \\ cjw@kth.se
}

\author{
P. Söderström \\ Vattenfall Distribution AB \\ Solna, Sweden
}

\author{
R. Saers \\ ABB Transformers \\ Ludvika, Sweden
}

\author{
O. Hansson \\ Fortum Distribution AB \\ Stockholm, Sweden
}

\begin{abstract}
Power system owners are facing major challenges, for example with changed electricity consumption and production patterns; more distributed generation and increased demand of cost efficiency while maintaining high reliability. The concept of dynamic rating can act as a part solution and implies that the capacity of a component is dynamically varying as a function of external parameters such as weather and load history. This hence implies that a component can be better utilized. This can have both direct economic benefits for the utility, but also benefits for the society and the environment by lower tariff levels, faster and cheaper connection of local environmentally friendly electricity production and less climate impact associated with component production and installation.
\end{abstract}

This paper gives a brief introduction to the concept of dynamic rating applied to power systems. Furthermore, results and conclusions from two workshops during 2013 are summarized, where representatives from Vattenfall and Fortum (distribution system operators), ABB (producer and developer that work with dynamic rating solutions), Swedish national grid and several persons from the academia contributed. This was complemented by interviews with the involved companies. The result is a mapping of knowledge, research, development interests, current situation and future visions. All parties show a great interest and see potential, but there are also challenges to be solved.

Keywords - adaptive rating; cable; control room; dynamic rating; management decision-making; power line; power system; standardization; thermal modeling; transformer; utalization; wind power

\section{INTRODUCTION}

Power system owners are facing major challenges with changed electricity consumption and production patterns; more distributed generation and increased demand of cost efficiency while maintaining high reliability. Structural refurbishments are hence needed to be increased in power systems to meet future requirements. Today, the capacity of power components often is set with static limits based on simulations of worst case scenarios. This leads to a discrepancy between the actuallyavailable capacity and the rating itself which unfortunately is left unused. [1] In order to use this unused capacity, it is important to formulate ratings on statically-set thermal limits and sags.

The approach of dynamic rating (DR) [2] implies that the capacity of a component is dynamically varying as a function of external parameters [3] [4] such as weather, load history and diagnostic indicators. The value of DR resides partly in utilizing existing equipment to a greater extent (used longer and/or more intense), and partly in the possibility of gaining better system performance (e.g. reliability) without passing loads which can lead to power outages, broken components or premature aging. This gives significant direct economic benefit by decreasing the need of costly investments. Furthermore DR also provides environmental benefits such as faster and less expensive connection of wind power and less environmental impact associated with component production.

DR is identified by several companies as an important future challenge and is e.g. proposed as a future research topic in a published report: "A vision on future research in electric power systems" [5]. Chapter II introduces some basic theory and exemplifies how capacity increment, net present value and reliability can be improved.

Chapter III-V mapping of knowledge, research, development interests, current situation and future visions concluded from workshops and interviews during 2013. These chapters are mainly based on following empirical materials:

- Workshop held in January 10, 2013 entitled: "DR Current situation, potential implementation and economic benefits". 16 participants from KTH Royal Institute of Technology (7), ABB (4), Vattenfall (3) and Swedish national grid (2).

- Workshop held in February 6, 2013 entitled: "DR Future Research and Development". 13 participants from KTH Royal Institute of Technology (8), ABB (2), Fortum (2), and Vattenfall (1).

\section{EXAMPLES OF POTENTIAL DYNAMIC RATING BENIFITS}

\section{A. Potential capacity increase using IEEE standard}

IEEE standard 738 [6] gives a standard formula for calculating the maximum current allowed $[A]$ of bare overhead conductors. It is based on steady-state heat balance:

$I_{\max }=\sqrt{\frac{q_{c}+q_{r}-q_{s}}{R\left(T_{C}\right)}}$

where $q_{c}[W / m]$ is the convective cooling, $q_{r}[W / m]$ is the radiative cooling, and $q_{s}[W / m]$ is the solar heating. $R\left(T_{C}\right)[\Omega / m]$ is the resistance of the conductor as a function of conductor temperature $T_{C}\left[{ }^{\circ} \mathrm{C}\right]$.

Reference [7] provides a simplified approximated formula on how the DR depends on temperature; for each decrease of one degree Celsius, the rating is assumed to increase with

Swedish Centre for Smart Grids and Energy Storage (SweGRIDS): http://www.kth.se/en/ees/omskolan/organisation/centra/swegrids 
$0.8 \%$ [7]. Other factors such as wind speed are not considered. This gives following formula:

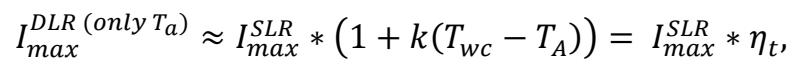

where $T_{w c}\left[{ }^{\circ} \mathrm{C}\right]$ is the temperature in the worst case scenario that the static rating is based on and $T_{A}\left[{ }^{\circ} \mathrm{C}\right]$ is the ambient temperature and $k\left[\frac{1}{{ }_{0}}\right]$ is a constant; in this case assumed to be equal to 0.08 based on [7]; $\eta_{t}$ is a non-dimensional scale function of how much the capacity is affected by temperature.

It is also of interest to investigate the impact of wind speed and how that may be integrated in the calculations. Especially, to handle wind power integration when high production correlates positively with increased line capacity. It can however be significant different wind characteristics between where the wind is measured and in the hot spot at a line corridor. This is a significant challenge to address when developing models relevant to use by utilizes within daily operation. In addition to $\eta_{t}, \eta_{v}$ is defined as a nondimensional scale function of how much the capacity is affected by wind speed and $\eta_{D L R}$ is defined as a nondimensional scale function of how much the capacity is affected by both temperature and wind speed.

The impact of wind speed is huge and nonlinear (higher impact when increasing from low wind speed compared to high speed. It is no significant differences between how the wind speed impacts at different ambient temperatures [9], i.e. if the wind speed differs while the temperature is set as constant, evaluated for different temperatures. Hence the contribution from wind speed and temperature can approximately be calculated separately and then added together, i.e.:

$$
I_{\text {max }}^{D L R}=I_{\text {max }}^{S L R} * \eta_{D L R} \approx I_{\text {max }}^{S L R} * \eta_{v} * \eta_{t}
$$

Worst case, that SLR is based on, is assumed when the wind speed is $0.5 \mathrm{~m} / \mathrm{s}$ as suggested in [8]. To derive an approximate formula of the wind contribution, the radial effect and solar heating (i.e. $q_{r}$ and $q_{s}$ ) are neglected, $R\left(T_{C}\right)$ is assumed to be constant and the wind angle is assumed to be perpendicular. By using IEEE standard 738, divide the formula with only wind speed $v\left[\frac{m}{s}\right]$ left unknown while temperature is assumed to be worst case and divide that with the same formula but with worst case wind speed, gives:

$I_{\max }^{D L R \text { only } v}=I_{\max }^{S L R} * \frac{I_{\text {max }}^{\text {DLR ony }}}{I_{\max }^{S L R}}=I_{\max }^{S L R} * \eta_{v}$

By simplifying as much as possible and implement more approximations, an approximate formula of the wind speed capacity contribution $\eta_{v}$ is provided in equation 5. Derivation, validation and more details of this formula are included in a paper submitted to IEEE Transactions on Smart Grid [9].

The measured wind speed is often too optimistic to use since in reality the wind speed is neither the same through the entire line nor perpendicular. The size of the scale parameter can be chosen based on where the wind speed data is measured (i.e. how similar characteristics at that place compared to the line corridor), the accuracy of the input data (e.g. how often the wind is measured), the height of the line and the surrounding landscape, but also how large other margins that exist and the chosen risk level.

$$
\eta_{v} \approx\left\{\begin{array}{c}
1 \quad \text { if } v_{\text {mod }} \leq 0.5 \mathrm{~m} / \mathrm{s} \\
1.20 * v_{\text {mod }}^{0.26} \text { if } v_{\text {mod }}=0.5-1.0 \mathrm{~m} / \mathrm{s} \\
1.20 * v_{\text {mod }}^{0.30} \text { if } v_{\text {mod }} \geq 1.0 \mathrm{~m} / \mathrm{s}
\end{array}\right.
$$

\section{B. Example of increased reliability example}

Assume a typical rural power distribution system, medium voltage, with two radial lines as illustrated in Figure 1, the dotted line excluded.

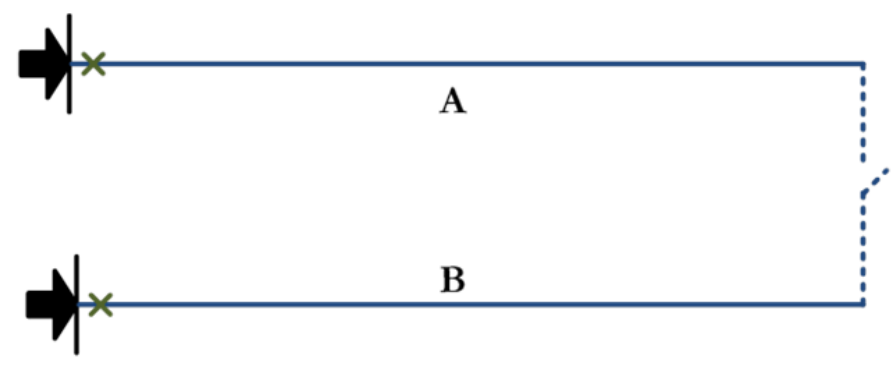

\section{Figure 1- Example of possible redundancy}

In Sweden for example, incentives that imply severe consequences of long outages have been introduced by laws [10]. An investment to mitigate the risk of long outages is hence motivated. One investment alternative is to replace overhead lines with cables which decrease the probability of long outages. However this is expensive and even if the failure rate decreases, the restoration time will not decrease.

Assume that it is possible to introduce reliability as shown by the dotted line in Figure 1 and that this is significant less expensive than other risk reducing investments. Assume however, that the load of all customers $(\mathrm{A}+\mathrm{B})$ could be higher that the static rating of current lines when it is cold in the winter. One solution is to introduce DR. In northern regions (as in Sweden), the highest load is in winter when it is cold which coincides with increased possible load limit if DR is used.

According to [10], the correlation between temperature and power consumption is close to $-1(\sim-0.90)$ and the correlation between wind speed and power consumption is close to zero $(\sim 0.03)$ in a power system situated in Sweden. If the temperature span is divided into separate analyses, it is possible to see that low wind speed both correlates with the highest and the lowest temperatures. However, power lines connected to wind power have of course a strong correlation between wind speed and line loading which can be advantageous, see section V.C.

\section{Net present value example}

Power system investments are often costly. If DR calculations results in that an investment can be avoided it provides direct savings. Even if the investment is postponed the 
economic savings can be significant. The net value saving is calculated as follows:

Saving $=C 1_{I}-\frac{C i_{I}}{\left(\frac{1+z}{100}\right)^{i}}$,

where $C 1_{I}$ is the investment cost today and $C i_{I}$ is the investment cost year $i ; Z[\%]$ is the internal rate of return and $i$ is number of years the investment can be postponed. For example, if an investment of $20000000 \mathrm{SEK}$ is postponed 10 years, the investment cost is assumed to be unchanged (i.e. $\left.C 1_{I}=C i_{I}\right)$ and the interest rate is assumed to be $7.5 \%$, the saving will be 10296121 SEK (or $51.5 \%$ ). Even if the investment is assumed to largely increase, it can still be significant savings to postpone. Even if the investment cost e.g. increases with $25 \%$ to 25000000 SEK in this example, it still gives a saving of 7870152 SEK. The cost of DR equipment and operation are not included here and will vary within different kind of implementations, but will probably decrease as the technique becomes more common and standardized. Still, these kind of cost calculation is of interest to define upper limits of what DR may cost today to implement based on commercial basis (in this example $\sim 10.3 / \sim 7.9$ million SEK), but also to motivate future DR R\&D.

\section{GENERAL DYNAMIC RATING R\&D ISSUES}

\section{A. Overview of the concept}

DR techniques can be used to operate electric power systems in a more efficient manner, without passing loads which can lead to power outages, broken components or hasty aging. In the future, with "Smart Grids" solutions and more distributed renewable energy sources, such as wind and solar, the benefits with DR will probably increase further. DR can best be described with the words "increasing the level of utilization."

DR is an interdisciplinary broad research area spanning from e.g. materials physics to social sciences. There is large interest in applied research, practical results and concrete company benefits. Vattenfall emphasized the importance that projects take a holistic approach, "going all the way." Important to include all cost aspects, to have a "win-win" approach for all stakeholders.

The conditions may look very different between countries and regions. Both in terms of current system margins and legal premises, but also and in terms of climate. Here countries with a cold climate have a benefit of the temperature part of DR since high demand often correlates with low temperatures. However the wind speed contribution has no positive correlation with high demand (when really cold, the wind speed tends to have an increased probability to be low).

\section{B. Dimensions of "dynamic rating level"}

One way of classification is to define three dimensions of "DR level":

1. Template levels, e.g. summer/winter: already often introduced.

2. Dynamic template levels based on weather forecast: a clear improvement compared with the first category for some categories of equipment.
However, it is doubtful whether this approach is applicable on components such as underground cables (see also Section IV.C), where the weather impact is slow and complicated.

3. Online measurements: often considered as expensive today. However, compared with e.g. invest in a new transformer it can probably be economic motivated, see section II.C . Sensors can detect weather conditions and/or component diagnosis such as sag or temperature. However, online measurements that can be used regarding DR already exist today. It is not always too expensive or unrealistic. Sensors used in online measurement must be easy to understand and not produce more input than relevant to handle.

Two other "dimensions" are to implement DR within new investment versus DR of existing equipment.

\section{Regulatory and legal incentives}

Power systems are considered as natural monopolies. The economic benefit for a utility is hence dependent of the regulation and laws in the country; whether better utilization of equipment is rewarded and how much.

In Sweden, the regulator has indicated the aim to increase incentives towards better power system utilization in the future.

\section{Decision support and increased acceptance}

DSOs responsible, they take the risk if problems occur, i.e. decisions on reducing margins intentionally must be based on well-argued materials.

There is preferable to use power distribution systems optimally and avoid over-investing. DR is a way to better utilize existing systems without increasing the risk too much. However, to be on the safe side, to trust the potential of DR and to take an active decision to invest less, this demands that such decisions rest on solid theoretic grounds. This motivates more research on risks and economic benefits associated with DR.

\section{E. How R\&D projects within DR should be designed}

There is a need of looking at "the big picture", that R\&D projects have a holistic perspective. All of following parts are equally important within a project: component modeling, information systems, financial aspects etc. There is a great value of conducting concrete case studies. Within 30 year DR and online measurement probably could be commonly used in power systems more generally.

In order to be able to reach long-term results within DR, successful short-term projects must be carried out. Depending on the business cycle and more, the trend is up and down regarding the acceptable length of a project until concrete results are reached. Now is the tendency towards short-term projects for DSOs. ABB has to always focus on both shortterm and more long-term $\mathrm{R} \& \mathrm{D}$ to handle future global competition.

There exist both general issues and company specific. Benefits of harmonization, general contracts etc. Special agreements in the industry should not be equated with unique agreement. A current problem is the need to standardize. 


\section{DYNAMIC RATING RELATED TO POWER COMPONENT}

\section{A. Components with most relevance}

According to the companies involved in this project: Power transformers, overhead lines and cables are probably of economic interest, other components are probably not economically justified. Protection equipment is also affected by temperature, but it is probably more about "optimizing" and introducing dynamic settings, i.e. "dynamic protection" of the three main component categories. The term "Adaptive Protection" is usually used when the protection settings (automatically) change depending on operating conditions or other external parameters

For the Swedish National Grid, the entire chain of components between two bus bars is of interest, i.e. the actual thermal line capacity. However, it is likely that other component limits do not vary as much as the capacity of overhead line and transformers, and hence may not have so "wide" DR possibilities. In an ideal world, the other components should be chosen so that they not restrict the line capacity; however, it should be noted that this is not always the fact due to different causes. An opinion is that the transformers invested in today are often too large; it is better to invest in smaller ones and have "controlled" overloading.

\section{B. Power transformers}

The transformer and its components, such as bushings and on-load tap changer, are one of the most critical parts in the electric power system due to the important function and the strategic location. At the moment, $\mathrm{ABB}$ has commercial products based on thermal models for transformers. Moreover ABB supports academic projects [11] and conducts a lot of own DR related R\&D. For example, a PhD project finished 2013 [12] has according to ABB produced a lot of innovative results.

$\mathrm{ABB}$ inserts the standard values in their provided models, but it is however up to the customer then to "increase the risk", set up equipment based on the model if they trust it. Often possible to increase the current permitted, but important measure that everything is okay before, i.e. no indications of e.g. higher temperature or gas oil as compared to normal. An approach is to comparing the measurements with the model, where large differences may indicate "something": If reality $\approx$ model $\rightarrow$ probably acceptable with "overloading".

Three temperatures are of interest: Top oil temperature, winding hot spot and other metallic temperature. One challenge is the difficulty to properly model the hot spot temperature. Temperature affects the permitted load of transformers. [13] [14] Adaptive protection and smarter cooling may also be of interest. To optimize cooling, start cooling earlier (based on e.g. forecasts). Offline and manual work is often time consuming and not reliable.

\section{Underground cables}

Underground cables have a lot of challenges regarding thermal modelling and are, as the case of power transformers, associated with high costs; both in terms of new investments and maintenance, but also if failures occurs. Since soil vary a lot regarding thermal properties, it is not possible to use same algorithms as for overhead lines. The thermal properties of soil are both depending on terrain and over time, e.g. as a result of the amount of water. Other challenges is the fact that the equipment is in the ground and hence difficult to inspect and troubleshoot and expensive to reach (especially in urban areas).

Old cables cause that the transmitted power must be reduced; DR can hence "be in both directions", i.e. also decrease limits compared with "normal" static ratings.

\section{Power lines}

For theoretical models of dynamic rating applied to power lines, see Section II.A.

\section{1) Transmission}

Swedish National Grid has a little DR today: line rates are constantly changing in the SCADA system, depending on the measured outside temperature. It exist a general interest and potential (technical) to develop current method such as algorithms, applications, methods etc.

\section{2) Distribution}

Only few parts affected by sag (before the rest of the line) and hence to focus on. The height could vary a lot along the same line. The terrain most important; identify most critical parts during worst case. Relatively gentle rules (sag); however, DSOs have often low margins today. Temperatures above $50^{\circ} \mathrm{C}$ is acceptable during shorter periods before sag, the critical temperature is about $70^{\circ} \mathrm{C}$.

See also Section V.C that addresses DR of power distribution lines to facilitate wind power integration.

\section{DYNAMIC RATING AT A SYSTEM LEVEL}

\section{A. Dynamic rating from a holistic perspective}

DR can be implemented in different parts of the system; the components that have been identified as the most relevant ones from an economic perspective are: transformers, overhead lines and cables, see Section IV.A. However, it is important to include safety equipment and to handle control rooms issues and looking at a system level. Both adaptive protection and control room issues have specifically been identified as important that need to be adapted in the future to manage more complex and dynamic solutions from a system perspective.

\section{B. Control room issues}

Some DR is already implemented on a limited scale today and will likely increase successively, both in extent of use and complexity. While the cost for online measurement gradually decreases and the technology improves, the control room has been identified as the enabler.

Today, the problem is not primarily to obtain large amounts of data, but to manage it. As the trend is towards more information to be handled in control rooms, it is not smart to have too many special solutions for the user interfaces and hence there exists a need to develop standard solutions and automation. Even if a DSO buy all solutions, it would be difficult to adjust processes and handle all information. It is also normal to use algorithms to handle data at "levels" before the control room instead, e.g. at the wind power plant. 
Another potential risk, that has to be investigated more, is that there always has been knowledge at the control rooms that an unused dynamic capacity often exist. Even if that not have been quantified or formalized, experienced control room personnel has, to some extent, used that knowledge as an "extra resource" if a disturbance occurs. This informal extra margin will decrease, the more DR is implemented. Probably, this is a possible risk only in some cases, but more research is needed.

\section{Dynamic rating to facilitate wind power integration}

It exist valuable positive correlation between high wind power production and weather that increase the transfer capacity, see Section II.A. Wind power rarely reaches its maximum production. By implementing DR and correlating the new ratings with wind power generation, the estimated time of "overloading" can be significantly reduced; for example, the probability is low that it is perfect wind conditions and extreme heat at the same time. This can remove or postpone some investment requirements. However, it also exist problems with that approach; for example, the wind speed is often lower at the power line compared with the rotor blades of a wind power plant. Furthermore, it is difficult to predict the wind speed in an entire line corridor, while windy.

Currently, both Vattenfall and Fortum conduct case studies to evaluate the use of DR for connecting wind power. Vattenfall held a presentation about an ongoing case study "Pilot Naum": Connecting wind power to a $40 \mathrm{kV}$ power distribution system, the introduction of DR and management to avoid overloads. A master project in collaboration between KTH and Fortum Distribution on DR and Wind Power connection has been completed [15]. That project evaluated a real case where wind power was connection to a $130 \mathrm{kV}$ power distribution system and show on significant benefits of using DR.

A further step is to design contracts where a DSO has the right to disconnect wind power in extreme cases, and in return compensate this by e.g. offering lower fees. Contract of permissible disconnection must consider several aspects: They must first of course comply with current legislation, and be beneficial to all parties. Another challenge is to define which stakeholder that is responsible for what and to define the burden of proof. There can be various companies involved, one that own the regional system, another that own the local system and several different ones that connect power production to the system. A contract has also to handle the disconnection prioritization when several different producers are connected.

The advantages of general contract templates would be: a) less legal costs if there are clear, elaborate contracts used by many, and b) that more companies would use such contracts if they existed, which would be both a benefit to the individual companies and even overall at an overall socio-economic level. Cheaper connection would also indirectly increase the share of renewable energy.

\section{Closure}

This paper gives a brief introduction to the concept of dynamic rating applied to power systems. The main contributions are mapping knowledge, research, development interests, current situation and future visions based on the perspectives from different stakeholders: utilities, producers and university.

The major conclusion is that it exist a significant potential and interest of DR from the perspectives of all stakeholders; that DR already is available to some extent, but will successively increase in the future. Furthermore it is important to investigate DR from a holistic perspective, taking all aspects into consideration. There are also a lot of challenges, for example the cost of collecting data decreases with time, while the focus has to more be of handling data in e.g. control rooms and develop general solutions.

Examples of some specific DR applications with a lot of research interest today are thermal models of power transformers and the usage of DR to facilitate wind power integration. Other identified issues of interest are for example "decision support and increased acceptance", "regulatory and legal incentives" and "standardization and harmonization".

\section{ACKNOWLEDGMENT}

Except the authors of this paper, following persons attended and contributed during 1-2 workshops (sorted alphabetically by first name): Arne Bergström (Vattenfall), Bengt-Olof Stenestam (ABB Components), Björn Weström (ABB Transformers), Christer Flood (Fortum Distribution), Johanna Rosenlind (KTH), Johan Söderbom (Vattenfall R\&D), Jurjen Kranenborg (ABB Corporate Research), Mattias Jonsson (SvK), Niklas Ekstedt (KTH), Per Westerlund (KTH), Rajeev Thottappillil (KTH), Saifal Talpur (KTH), Sajeesh Babu (KTH), Samir Lodhi (ABB Ventyx), Sebastian Svedberg (KTH) and Wissam El Marakbi (SvK).

In addition Jan Henning Jürgensen (KTH) contributed with proofreading.

\section{REFERENCES}

[1] H. Wan, J. McCalley, and V. Vittal, "Increasing thermal rating by risk analysis," IEEE Transactions on Power Systems, vol. 14, pp. 815 - 828, Aug. 1999.

[2] D. A. Douglass and A. Edris, "Real-time monitoring and dynamic thermal rating of power transmission circuits", IEEE Transactions on Power Delivery, vol. 11, issue 3, pp- 1407-1418, May-June 1996.

[3] P. Reding, "A method for determining probability based allowable current ratings for bpa's transmission lines," IEEE Transactions on Power Delivery, vol. 9, pp. 153 - 61, Jan. 1994.

[4] C. Price and R. Gibbon, "Statistical approach to thermal rating of overhead lines for power transmission and distribution," IEE Proceedings on Generation, Transmission and Distribution, vol. 130, pp. $245-156$, Sep. 1983.

[5] M. Bollen, "A vision on future research in electric power systems", Luleå University of Technology, Electric Power Engineering, May 2012

[6] IEEE, "Standard for calculating the current-temperature of bare overhead conductors," IEEE Std 738-2006

[7] D. Douglas, "Dynamic Rating of Overhead Lines", IEEE Tutorial, Detroit, MI, July 25, 2011: http://ieee-tpc.org/ieee tutorials/IEEETutorial_DynamicthermalLineRatings_26July2011.pdf

[8] E. N. A. (ENA), "Engineering recommendation p27-current rating guide for high voltage overhead lines operationg in the uk distribution system," 1986.

[9] C. J. Wallnerström, Y. Huang and L. Söder, " Impact from Dynamic Line Rating on Wind Power Integration into Distribution Power Systems", submitted (29 Nov 2013) to IEEE Transactions on Smart Grid. 
[10] C. J. Wallnerström and P. Hilber, "Vulnerability Analysis of Power Distribution Systems for Cost-effective Resource Allocation," IEEE Transactions on Power Systems, vol. 27, pp. 224 - 232, Feb. 2012.

[11] J. Rosenlind and P. Hilber, "On improvements of conditions monitoring of transformers by considering thermal models," in IEEE 12th International Conference on Probabilistic Methods Applied to Power Systems (PMAPS), Jun. 2012.

[12] J. Rosenlind, "Lifetime modeling and management of transformers", $\mathrm{PhD}$ Thesis, KTH, Sweden, October 2013.
[13] M. F. Lachman, P. J. Griffin, W. Walter, and A. Wilson, "Real-time dynamic loading and thermal diagnostic of power transformers," IEEE Transactions on Power Delivery, vol. 18, pp. 142 - 148, Jan. 2003.

[14] D. Susa and M. Lehtonen, "Dynamic thermal modeling of power transformers: Further development - part 1," IEEE Transactions on Power Delivery, vol. 21, pp. 1961 - 1970, Oct. 2006.

[15] Saifal Talpur, "Dynamic line rating implementation as an approach to handle wind power integration - A feasibility analysis in a subtransmission system owned by Fortum Distribution AB”, M.Sc. Thesis, KTH, 2013 